\title{
VIOLENCIA DE GÉNERO CONTRA LAS MUJERES EN AMÉRICA LATINA: UNA REFLEXIÓN CRÍTICA EN TORNO A LAS AGENDAS DE GÉNERO EN LA INTEGRACIÓN REGIONAL. LOS CASOS DE MERCOSUR Y SICA
}

\section{Gender-based violence against woman in Latin America: a critical reflection on gender agendas in regional integration. The cases of MERCOSUR and SICA}

\author{
Fabiana Rivas Monje \\ Malena Zunino Folle
}

\section{RESUMEN}

Si bien los procesos de integración regional en América Latina se remontan a mediados del Siglo XX, sus objetivos y lineamientos se han modificado mediante múltiples mecanismos de actualización de sus directrices. No obstante, la violencia de género contra las mujeres sigue ubicada en los márgenes de las agendas regionales. En consecuencia, no se ha mitigado su impacto en la región, más aun, América Latina se configura actualmente como el territorio más peligroso para las mujeres. Así, el objetivo del artículo pretende reflexionar desde un abordaje teórico, en torno a la incorporación de la agenda de género a los procesos de integración regional latinoamericana, enfocado en la violencia contra las mujeres. Analizaremos dos casos particulares: el Mercado Común del Sur y el Sistema para la Integración Centroamericana y, posteriormente, trabajaremos con estadísticas y legislaciones de sus países miembros para esbozar lineamientos finales a través de planteamientos críticos y problematizadores. Es menester considerar y repensar nuevos mecanismos para ampliar y fortalecer la lucha contra las múltiples manifestaciones que violentan los cuerpos de las mujeres y superar enfoques localistas en pos de apuntar a objetivos colectivos contra este fenómeno que atraviesa todas las sociedades del mundo. De esta manera, la integración regional aparece como una herramienta con importantes potencialidades que urge reconsiderar.

PALABRAS CLAVE

Violencia de género contra las mujeres; Agenda de género; MERCOSUR; SICA

\begin{abstract}
Although regional integration processes in Latin America date back to the mid-twentieth century, their objectives and guidelines have been modified through multiple mechanisms in order to update their guidelines. However, gender-based violence against women continues to be located at margins of regional agendas. Therefore, its impact in the region hasn't been mitigated, moreover, Latin America is currently the most dangerous territory for women. Consequently, the purpose of this document is to reflect from a theoretical approach, on the incorporation of the gender agenda in the processes of Latin American regional integration, particularly focused on violence against women. We will focus on the analysis of two particular cases: the Common Market of the South and the Central American Integration System. Subsequently, we will work with statistics and legislation of MERCOSUR and SICA member states, to outline final guidelines through critical and problematic approaches. It's necessary to consider and rethink new mechanisms to expand and strengthen the fight against the multiple manifestations that violate women's bodies, to overcome local approaches in order to reach
\end{abstract}


Violencia de género contra las mujeres en América Latina: una reflexión crítica en torno a las agendas de género en la integración regional. Los casos MERCOSUR y SICA DOI: https://doi.org/10.24215/24689912e022

collective solutions against this phenomenon that affects all societies around the world. In this regard, regional integration stands as a tool with significant potential that needs to be urgently reconsidered.

\section{KEY WORDS}

Gender violence against women; Gender agenda; MERCOSUR; SICA 


\section{INTRODUCCIÓN}

El objetivo del presente documento pretende reflexionar en torno a la incorporación de la agenda de género a los procesos de integración regional latinoamericana, particularmente enfocada en la violencia contra las mujeres. Para ello, nos abocaremos al análisis de dos casos particulares: el Mercado Común del Sur $^{1}$ y el Sistema para la Integración Centroamericana ${ }^{2}$. Cabe aclarar que no se intenciona un análisis comparativo, en tanto las realidades regionales difieren considerablemente entre una y otra y, en consecuencia, sus particularidades le imprimen características específicas. Sin embargo, como se constatará en las siguientes páginas, ello no supone la inexistencia de ciertas similitudes y caminos compartidos respecto a la construcción de la integración. Vincular la integración regional con el enfoque de género es, no sólo vital sino imperante por varios motivos. En primer lugar, enriquece y complejiza el estudio integracionista al incorporar una problemática tan cotidiana como dolorosa que nos atraviesa e interpela como habitantes de la región más insegura para las mujeres del mundo, en la cual diariamente doce mujeres son asesinadas, es decir, donde cada tres horas se comete un femicidio.

Como se verá más adelante, los mecanismos de integración han realizado esfuerzos en actualizar sus plataformas a través de la incorporación de demandas y actores nogubernamentales que se enmarcan en sus respectivos procesos de relanzamiento. Así, hacer dialogar a la integración regional con las agendas de género permite desentrañar progresivamente la concepción abstracta que reviste a los mecanismos de integración en la región, generalmente asociados exclusivamente a niveles institucionales y estatales, en definitiva, escindidos de las realidades cotidianas de las y los latinoamericanas/os.

Por último, esta propuesta cobra especial relevancia en la coyuntura actual donde las demandas y acciones por los derechos de las mujeres han ido tomando progresivamente formas regionales, mediante procesos colectivos que superan las fronteras nacionales. Este fenómeno se ve reflejado, por ejemplo, en el movimiento $\mathrm{Ni}$ Una Menos que, a pesar de haber surgido en Argentina en 2015, ya para los siguientes años se replicó en varios países de la región (Ferretto \& Picasso, 2018) o

\footnotetext{
${ }^{1}$ En adelante MERCOSUR

${ }^{2}$ En adelante SICA
} 
en la Campaña Nacional por el Aborto Legal cuya mayor expresión también tuvo lugar en Argentina en 2018, y ese mismo año varios países se unieron a las demandas, llevando a cabo acciones propias en sus contextos nacionales ${ }^{3}$.

Las páginas siguientes se organizan en tres apartados. En primer lugar, se plantea una revisión teórica en relación con los periodos de integración regional en Latinoamérica, en función de encuadrar temporal y espacialmente al MERCOSUR y SICA. Ello se aborda bajo el entendido de que la integración regional como categoría requiere ser explicada constantemente ${ }^{4}$, al igual que sus configuraciones y contenidos. En este sentido, el concepto de "coeficiente histórico" de Ansaldi (2013) guía el análisis y habilita la incorporación de la perspectiva de larga duración de la integración, a través del enlace de líneas de continuidad y de ruptura que configuran experiencias concretas en coyunturas determinadas.

En segundo lugar, se presentan ambos modelos de integración para dar paso a la manera en que estos integran una agenda de género, particularmente en su abordaje de la erradicación de la violencia contra las mujeres.

Por último, a través de la exposición de estadísticas y legislaciones en los países miembros del MERCOSUR y SICA, se elaboran las reflexiones finales desde una mirada integral a través de planteamientos críticos y problematizadores en relación con lo expuesto.

Convencidas de que es necesario eliminar todas las manifestaciones de violencia hacia las mujeres, en todos los niveles y ámbitos de existencia, entendemos que la integración regional es un mecanismo clave para su erradicación definitiva y, como tal, no puede permanecer ajeno a este flagelo. De esta manera y, a través de las próximas páginas, asumimos y reafirmamos nuestro compromiso como mujeres de las ciencias sociales para superar el sesgo comercial y economicista con que suele abordarse esta temática.

\footnotetext{
${ }^{3}$ Como es el caso de Brasil, Chile, Colombia, Costa Rica, Ecuador, México, Paraguay y Perú, donde las articulaciones feministas tomaron más fuerza luego del 13J (13 de julio de 2018). Recuperado de: http://latfem.org/aborto-legal-en-argentina-aborto-legal-en-america-latina/

${ }^{4}$ Reflexión tomada a partir de los aportes vertidos en la asignatura "Los procesos de integración en América Latina", a cargo del docente Alejandro Frenkel, inscrita en el programa académico de la Maestría en Estudios Sociales Latinoamericanos (MESLA-UBA).
} 


\section{ALGUNAS CONSIDERACIONES PRELIMINARES SOBRE LA INTEGRACIÓN REGIONAL}

La literatura avocada en la materia de integración regional, identifica tres oleadas/periodos según sus configuraciones particulares. El primer periodo se relaciona con la constitución de la Unión Europea a partir de 1950, donde surge la idea de cooperación económica y es liderada por los expertos en la materia y reformistas políticos latinoamericanos (Wionczek, 1970). Así, con la influencia de los aportes teóricos provistos por la CEPAL, se entendió la integración económica como un factor de desarrollo, en tanto estimularía la modificación del modelo de exportación tradicional y, en consecuencia, modernizaría las economías de la región (Wionczek, 1970). Por otra parte, se vio en la integración un potencial para mitigar la brecha entre estos países y las potencias mundiales del Norte, lo cual se planteó a través de objetivos y lineamientos que reflejaban la concepción unidimensional imperante en aquel entonces (como el caso de $\operatorname{ALALC}^{5}$ y $\mathrm{MCC}^{6}$ ).

El segundo periodo se vincula a la proliferación de bloques regionales enmarcados en la globalización de los 90 (por ejemplo, el caso del MERCOSUR y la reestructuración del MCC en SICA). En este sentido, las palabras de Van Klavaren (1997) quien, citando a Gert Rosenthal (1991) echa luz al respecto:

Si en el pasado la integración fue concebida como un instrumento de defensa colectiva contra las adversidades originadas en el sector externo, hoy tiende a ser concebida como un elemento más bien ofensivo y flexible que contribuye a mejorar la inserción internacional de América Latina. (Van Klavaren, 1997, p.53)

Finalmente, la tercera oleada se presenta como propia de América Latina (por ejemplo UNASUR ${ }^{7}$ ) también llamada regionalismo postliberal (Veiga \& Ríos, 2007; Sanahuja, 2010, citados por Caballero, 2017). La misma se caracteriza por una ampliación de agendas y una cooperación más "política" que la anterior (Fawcett \& Hurrell, 1994;

\footnotetext{
${ }^{5}$ La Asociación Latinoamericana del Comercio (ALCA), se integró por (1960-1980). Integrado por: Argentina, Brasil, Chile, Paraguay, Uruguay y México (1960 - 1980)

5 El Mercado Común Centroamericano (MCC), se integra por: Guatemala, El Salvador, Honduras, Nicaragua, Costa Rica y Panamá.

${ }^{7}$ La Unión de Naciones Suramericanas (UNASUR), estuvo inicialmente integrada por los doce países de la Región suramericana: Argentina, Bolivia, Brasil, Colombia, Chile, Ecuador, Guyana, Paraguay, Perú, Suriname, Uruguay y Venezuela.
} 
Ruiz, 2006; Bulmer, 2007; Dabène, 2012; Botto, 2015, citados por Ferretto \& Picasso, 2018). Bajo estos entendidos, se apostó al fortalecimiento del rol estatal frente al mercado y a la ampliación de agendas sociales ante las brechas e inequidades existentes en la región, mediante la integración de otros actores no estatales y de la sociedad civil (Caballero, 2017).

Por último, y siguiendo con los aportes de Caballero (2017), es menester agregar que la coyuntura actual implica cuestionar la continuación del periodo anterior $y$, en consecuencia, la existencia de un posible nuevo periodo en la integración regional latinoamericana.

\section{BREVE CONTEXTUALIZACIÓN Y ANTECEDENTES DE LOS BLOQUES DE INTEGRACIÓN REGIONAL LATINOAMERICANA: LOS CASOS DE MERCOSUR Y SICA}

EI MERCOSUR fue establecido en 1991 mediante el Tratado de Asunción, firmado por Argentina, Brasil, Paraguay y Uruguay ${ }^{8}$. Para 1994 con la firma del Protocolo Adicional a través del Protocolo de Ouro Preto, se establece la estructura institucional definitiva ${ }^{9}$. Siguiendo a Malamud (2011), además de la complementariedad y las economías de escala, el objetivo inicial era la reducción de amenazas a las democracias nacientes mediante la desactivación de hipótesis de conflicto que alimentasen los presupuestos y fobias militares, aunque el articulado contemplara inicialmente objetivos económicos y comerciales a fin de consolidar un mercado común.

El agotamiento del modelo neoliberal trajo consigo una reconfiguración en los presupuestos del bloque, sea por el ascenso de gobiernos denominados progresistas en la región y/o por los nuevos paradigmas imperantes en dichos países. A partir del nuevo milenio MERCOSUR se relanza e inicia una nueva etapa conocida como el MERCOSUR Social y Productivo (Briceño Ruiz, 2011, citado por Ferretto \& Picasso, 2018), caracterizada por la contemplación de agendas sociales y políticas de cooperación productiva. En línea con los principios de Democracia y Desarrollo

\footnotetext{
${ }^{8}$ Posteriormente se incorporaron la República Bolivariana de Venezuela, que se encuentra actualmente suspendida de todos sus derechos y obligaciones (conforme a lo dispuesto en Art. 5, Protocolo de Ushuaia), y el Estado Plurinacional de Bolivia, que se encuentra en proceso de adhesión. Chile, Colombia, Ecuador, Perú, Guyana y Surinam integran el bloque como Estados Asociados.

${ }^{9}$ Compuesta por tres órganos decisorios y tres no decisorios.
} 
Económico, se busca impulsar una "integración con rostro humano"10, a través de diferentes acuerdos en materias migratoria, laboral, social y cultural. Aquí tiene cabida realizar el nexo con la proliferación de nuevas agendas sociales, donde las demandas de equidad e igualdad de género forman parte.

Por otro lado, la región centroamericana, en el marco de las transformaciones constitucionales internas y los procesos de consolidaciones democráticas, firma el Protocolo de Tegucigalpa del año 1991 en la XI Reunión de Presidentes, donde se consolida el SICA, con la adhesión de El Salvador, Guatemala, Nicaragua, Costa Rica, Honduras y Panamá ${ }^{11}$. Tomando como antecedentes experiencias previas de integración en la región (MCC), el Art. 1 del Protocolo de Tegucigalpa define que el objetivo fundamental del SICA es la realización de la integración centroamericana, para constituirla en una región de paz, libertad, democracia y desarrollo (Secretaría General del Sistema de la Integración Centroamericana, 2008). Entre sus principios, se alinea a la normativa internacional emanada del Sistema Universal Interamericano de Derechos Humanos, que propulsaba la erradicación de toda forma de discriminación y violencia contra las mujeres (Letona, 2017). En la Reunión Extraordinaria de Jefes de Estado y de Gobierno (2010) y, por iniciativa de El Salvador y Guatemala, se elabora y posteriormente aprueba la agenda del 'Relanzamiento de la Integración Centroamericana'. Este impulso se basó en cinco ejes sectoriales: seguridad democrática, prevención y mitigación de desastres naturales y efectos del cambio climático, integración social, integración económica y fortalecimiento institucional. Ello implica la integración cada vez mayor de la sociedad civil y enfatiza en el propósito de una mayor solidez regional (Sánchez, 2017). Más aún, esta agenda ha conseguido articular un catálogo de acciones de política regional que supera los límites de la integración económica y retoma el compromiso inicial con el desarrollo (Santos y Pozo, 2013; Caldentey, 2014, citados en Santos Carrillo, 2016).

Así, se puede observar cómo ambos bloques regionales buscan ampliar sus marcos de acción comercial y económica. Coincidente con el período del regionalismo posliberal, tanto el MERCOSUR como el SICA a través de sus renovaciones y

\footnotetext{
${ }^{10}$ Sitio Web oficial MERCOSUR: https://www.mercosur.int/quienes-somos/en-pocas-palabras/

${ }_{11}$ Posteriormente se adhirieron como miembros plenos Belice en el año $2000 \mathrm{y}$, a partir de 2013, la República Dominicana.
} 
relanzamientos se abocaron a la multidimensionalidad de la integración, formulando nuevas directrices y reformulando sus agendas alineadas a los tratados internacionales en referencia a los Derechos Humanos y, por consiguiente, abordando las desigualdades de género y la violencia contra las mujeres.

\section{AGENDA DE GÉNERO EN EL MERCOSUR}

Ya para los años 80 se buscaba a nivel internacional la incorporación transversal de la categoría género en las políticas públicas, a fin de impactar en los procesos de definición, ejecución y evaluación de éstas, superando la consideración del género como una problemática aislada o aparte, y la subsecuente elaboración de programas y políticas enfocados en mujeres (Gómez \& Naclerio, 2011). Así, para la época que tuvo lugar al proceso histórico-político de constitución del MERCOSUR, el contexto mundial de los derechos de las mujeres estaba marcando la agenda (Ferretto \& Picasso, 2018). En este sentido, en el ámbito del MERCOSUR y desde su constitución, las agrupaciones de mujeres y feministas, así como ONG y organizaciones como el Fondo de Desarrollo de las Naciones Unidas para la Mujer -UNIFEM- y la Facultad Latinoamericana de Ciencias Sociales -FLACSO-, aunaron esfuerzos para introducir la consideración de la dimensión de género en el proceso de integración y cooperación regional (Gómez \& Naclerio, 2011), puesto que como se ha expuesto, desde sus comienzos los debates principalmente giraron en torno a cuestiones de carácter económico, comercial y político.

El contexto internacional respecto a las cuestiones de género que repercutieron en el proceso mismo de constitución y evolución de estos temas en el MERCOSUR, está marcado a partir de 1975 principalmente por instancias como las Conferencias Mundiales en el marco de acción de la ONU ${ }^{12}$ y la "década de la mujer" (1975-1985), donde los acuerdos que surgieron marcaron ocasiones de encuentro, comunicación y acción coordinada, y donde la escala de acción de las mujeres fue múltiple: el escenario mundial en el marco de ONU, el horizonte regional de América Latina y los

${ }^{12}$ En 1975 se realiza la I Conferencia en México, 1980 en Copenhague, 1985 en Nairobi, y la última en Beijing 1995, donde se aprobó la Plataforma de Acción de Beijing. Ulteriormente, se llevaron a cabo revisiones a través de los Seguimientos Beijing +5 en 2000, Beijing +10 en 2005, y Beijing +15 en 2010 . 
planos nacionales, así como provinciales y $\operatorname{locales}^{13}$ (Jelin, 2002).

En este sentido, el proceso regional del MERCOSUR respondió principalmente a los postulados de la Convención Sobre la Eliminación de Todas las Formas de Discriminación contra la Mujer (CEDAW, por sus siglas en inglés) de 1979, la Declaración y Plataforma de Acción de Beijing de 1995 y la Convención Interamericana para Prevenir, Sancionar y Erradicar la Violencia contra la Mujer, así como los Consensos de Quito (2007), Brasilia (2010) y Santo Domingo (2013), asumidos en el marco de las Conferencias Regionales sobre la Mujer de la Comisión Económica para América Latina (CEPAL).

En 1995 se creó la red Foro de Mujeres ${ }^{14}$ del MERCOSUR (que funcionó hasta 2005), y en julio de 1998 y, a través de la resolución №20/98, se creó la Reunión Especializada de la Mujer -REM-, en el marco de la Reunión del Grupo de Mercado Común -GMC-, cuyo objetivo era "analizar la situación de la mujer, teniendo en cuenta la legislación vigente en los Estados Partes del MERCOSUR, en lo relativo al concepto de igualdad de oportunidades, con el fin de contribuir al desarrollo social, económico y cultural de las comunidades" ${ }^{\prime 1}$. Para 2011, se generó un nuevo hito en la institucionalidad de género del MERCOSUR: teniendo en cuenta los avances institucionales de los organismos nacionales de políticas para las mujeres, la REM solicitó la elevación de su estatus a Reunión de Ministras y Altas Autoridades de la Mujer -RMAAM ${ }^{16}$-, cuya función principal es asesorar y proponer al Consejo del Mercado Común (CMC), medidas, políticas y acciones en materias de igualdad de género.

${ }^{13}$ El horizonte latinoamericano fue un eje organizador privilegiado en esta etapa: en 1981 se comenzaron a realizar Encuentros Feministas de América Latina y a gestar redes latinoamericanas ligadas a temas específicos, como la Red de Salud de Mujeres Latinoamericanas y del Caribe, la Red de Educación Popular entre Mujeres, la Red Latinoamericana contra la Violencia Doméstica y Sexual, y el Consejo Latinoamericano de los Derechos de la Mujer (Jelin, 2002).

${ }^{14}$ Participó en la creación de REM y como asesor de ésta hasta 2005. Este foro implementó los Encuentros Regionales de mujeres realizados en diferentes ciudades de los países del MERCOSUR, propiciando la participación de mujeres políticas, trabajadoras, empresarias, profesionales y educadoras, dando legitimidad a las propuestas realizadas en cada reunión de REM (Gómez y Naclerio, 2011).

${ }_{15}$ Art. $1^{\circ}$ de la Resolución 20/98 del GMC. En: http://www.observatoriomercosur.org.uy/es/obsd33.php.

${ }^{\frac{16}{16}}$ Integrada por representantes gubernamentales de los Estados Parte. 
Actualmente la RMAAM es el principal foro de coordinación política entre las máximas autoridades de los mecanismos para el adelanto de la mujer, dirigida a promover y facilitar la generación de condiciones para un ejercicio pleno de los derechos de las mujeres, donde se debate, promueve y facilita la definición de políticas públicas regionales para las mujeres y la igualdad de género. (RMAAM, 2014, 13)

En 2014, la RMAAM formula la Política de Igualdad de Género del MERCOSUR, documento producto del Encuentro sobre Lineamientos de Política de Igualdad de Género en el MERCOSUR realizado en 2013, con el objetivo de implementarla a través de puntos focales de los órganos y foros del organismo. Las Directrices de dicha política, -constituida por trece ítems temáticos- subrayan la necesidad de la integración del género en el conjunto de políticas y estructura institucional del MERCOSUR en la dimensión institucional y la de políticas regionales.

Respecto a la problemática de la violencia contra las mujeres, las Directrices manifiestan en su punto 6.7. "Enfrentar todas las formas de violencia basada en la perspectiva de género", a través de la adopción de todas las medidas necesarias para prevenir, atender, erradicar, sancionar y reparar las diversas formas de violencia contra las mujeres, como la física, sexual, patrimonial, económica, psicológica, simbólica que menoscaben el ejercicio de los derechos y dignidad de las mujeres, incluyendo el femicidio/feminicidio y la trata, observando a la violencia contra las mujeres como una violación a los DDHH (RMAAM, 2014).

La implementación de la política es responsabilidad de todos los órganos del MERCOSUR, mediante estrategias que incluyan acciones específicas como su incidencia sobre los puntos focales de órganos y foros, el desarrollo de información y estadísticas rigurosas, presentación de informes anuales al CMC del estado de avance y la elaboración de un plan de acción para la promoción de la igualdad de género en el MERCOSUR en sus dimensiones institucional y de políticas regionales (RMAAM, 2014). El monitoreo y seguimiento de las distintas etapas de aplicación es responsabilidad de la RMAAM.

\section{Agenda de GÉnero En EL SICA}

Al ampararse en la normativa internacional emanada del Sistema Universal 
Interamericano de Derechos Humanos, en el SICA se constata que todos sus Estados Parte han firmado y ratificado -al igual que MERCOSUR- los marcos de la CEDAW, la Plataforma de Acción de Beijing y Belém do Pará. En consecuencia, el encuadre jurídico y político del SICA reconoce la violencia contra las mujeres como una problemática de seguridad. En función de ello, se han establecido compromisos -tanto regionales como internacionales- y diseñado estrategias con el objetivo de su erradicación, cuyos esfuerzos quedan plasmados fundamentalmente a través de tres grandes hitos: el Tratado Marco de Seguridad Democrática de 1995, la incorporación del Consejo de Ministras de la Mujer de Centroamérica y República Dominicana (COMMCA) al SICA (2005), y la creación de la Política Regional de Igualdad de Género, PRIEG (creado en el 2013, pero actualizado en el 2018).

El Tratado Marco de Seguridad Democrática de 1995 actúa como instrumento complementario al Tratado de Tegucigalpa a través de la creación de una plataforma jurídica, donde se impulsa un desarrollo integral de cara al nuevo modelo de seguridad democrática, en tanto refleja la necesidad de las democracias latinoamericanas en aquel entonces, "de definir sus roles, y posicionarse con derecho propio en el concierto de las naciones del mundo" (Blanco, 2012). De esta manera, reafirman el compromiso con la democracia:

Se sustenta en la supremacía y el fortalecimiento del poder civil, el balance razonable de fuerzas, la seguridad de las personas y de sus bienes, la superación de la pobreza y de la pobreza extrema, la promoción del desarrollo sostenible, la protección del medio ambiente, la erradicación de la violencia, la corrupción, la impunidad, el terrorismo, la narcoactividad, el tráfico de armas (Tratado Marco de Seguridad Democrática, 1995) ${ }^{17}$.

Aquí no se hace referencia explícita a la violencia contra la mujer, lo que se explica por el contexto temporal y regional, dejando entrever la incidencia que los conflictos armados han tenido en la región y cómo las múltiples maneras de violencia se fueron tejiendo a través de un complejo entramado.

17 Art. 1 del Tratado Marco de Seguridad Democrática de 1995. En: https://www.iidh.ed.cr/multic/UserFiles/Biblioteca/IIDHSeguridad/12 2010/4b294167-d00a48048336-0a6739d1e0a6.pdf 
En el año 2005, la XXVI Reunión de Jefes de Estado y de Gobierno y, fruto de diversas instancias que comenzaron con la VI Cumbre Iberoamericana de Jefes de Estado y de Gobierno en el año 2000, el SICA integró bajo la órbita de la Secretaría General (SG-SICA) a la Secretaría Ejecutiva del COMMCA, la cual se constituye como el órgano político superior en lo referido a la equidad y a la igualdad de género del SICA. En función de incidir en cambios necesarios para que las mujeres avanzaran en el ejercicio de su ciudadanía y de Derechos Humanos y con una mirada integral del desarrollo social, político y económico de la mujer, se estableció un trabajo articulado de la Secretaría Técnica de la Mujer- COMMCA cuyo objetivo principal es:

Promover propuestas de políticas en el ámbito regional orientadas a transformar la condición, situación y posición de las mujeres de la región y a la adopción de una política y estrategia sostenible de equidad de género propiciando el desarrollo de los países que integran el SICA ${ }^{18}$.

Esta acción coordinada entre el COMMCA y los demás órganos del SICA estableció la necesidad de crear nuevos procesos de institucionalización, seguimiento y monitoreo (Reglamento de organización y funcionamiento del COMMCA, 2011, 7), en pos de transformar la situación de desigualdad de las mujeres en la región y así alcanzar un mejor nivel de desarrollo y una mejor calidad de vida de sus ciudadanos y ciudadanas $^{19}$. Asimismo, le compete el análisis, diagnóstico, elaboración de propuestas y recomendaciones dirigidas a promover, vincular, integrar y posicionar el desarrollo de los Derechos Humanos universales de las mujeres (Letona, 2017). Entre sus objetivos específicos, el que refiere a violencia de género estipula la eliminación de las prácticas de violencia en general, violencia doméstica e intrafamiliar y trata de personas para explotación sexual.

Sus objetivos son planteados a través de estrategias a mediano plazo; en este sentido en el segundo de sus Planes Estratégicos (2009-2013) priorizó como eje transversal la Violencia contra las Mujeres.

Es mediante la incorporación del COMMCA que comenzó una estrategia, aún vigente,

\footnotetext{
${ }^{18}$ Sitio Web oficial SICA: https://www.sica.int/commca/breve.aspx

${ }^{19}$ Convenio Constitutivo del Consejo de Ministras de la Mujer de Centroamérica (COMMCA) viernes, 12 de agosto de 2005.
} 
de transversalización de género en los instrumentos políticos y en todos los niveles de la institucionalidad del SICA (Letona, 2017). En este marco se diseñó -con posterior aprobación en la Reunión de Presidentes en el marco de la XLII Reunión Ordinaria del 14 de diciembre del 2013- la Política Regional de Igualdad de Género (PRIEG). La misma pretende accionar como una plataforma común a través de 62 medidas, que busca la integración de las temáticas de género a las demás agendas políticas de los Estados miembros del SICA. Mediante una política a largo plazo con miras hacia el 2025 se plantea que los Estados parte del hayan:

Incorporado las medidas necesarias para garantizar el pleno desarrollo y el adelanto de las mujeres de Centroamérica y República Dominicana, en condiciones de igualdad y equidad, en la esfera política, social, económica, cultural, ambiental e institucional, tanto a escala regional como en los ámbitos nacionales. (STM-COMMCA/SICA, 2014)

De su resolución se desprenden 8 lineamientos; entre ellos, el $\mathrm{N}^{\circ} 6$ hace referencia directa a la violencia contra la mujer, proponiendo: "Incorporar en la agenda de seguridad democrática de la región, el enfoque de seguridad ciudadana de las mujeres fortaleciendo las políticas y programas dirigidos a prevenir, detectar, atender, sancionar y erradicar la violencia contra las mujeres y de género en la región"20.

Destaca de su diseño una perspectiva transversal, multidimensional y multisectorial, que apuesta en tanto construcción colectiva a una "intervención colectiva entre los países miembros para impulsar cambios intersectoriales y a nivel regional"21; así se traza la necesidad de establecer un compromiso que articule la coordinación tanto a nivel nacional como regional.

\section{Qué hace cada Estado PARTE En RELACIÓN CON EL MARCo NORMATIVO de} MERCOSUR Y SICA: ESTADÍSTICAS Y LEGISLACIONES VIGENTES EN MATERIA DE VIOLENCIA CONTRA LAS MUJERES

20 Art. 6 del PRIEG. En: http://genero.ccj.org.ni/publicaciones\%20general\%20$\% 20 \mathrm{CCJ} /$ Internacionales/5Politica $\% 20$ Regional $\% 20 \mathrm{de} \% 20$ lgualdad $\% 20 \mathrm{y} \% 20$ Equidad $\% 20 \mathrm{de} \%$ 20Genero\%20\%28PRIEG\%29.pdf

21 Art. 6 del PRIEG. En: http://genero.ccj.org.ni/publicaciones\%20general\%20$\% 20 \mathrm{CCJ} /$ Internacionales/5Politica $\% 20$ Regional\%20de $\% 20$ lgualdad $\% 20 \mathrm{y} \% 20$ Equidad $\% 20 \mathrm{de} \%$ 20Genero\%20\%28PRIEG\%29.pdf 
En lo referido al accionar concreto a nivel institucional y de políticas públicas en torno a la violencia contra las mujeres, sobre finales de la primera década del presente siglo los Estados Parte de ambos bloques, MERCOSUR y SICA, han respondido con legislaciones y creación de normativas e instrumentos respecto a la problemática. En este aspecto, cabe señalar que los mecanismos regionales, así como internacionales, promueven la creación de legislaciones específicas y reformas de los Códigos Penales de cada país en particular, reflejando un entendimiento integral de la violencia. Si bien se han dado procesos diversos en cada país, se puede observar cómo a partir del nuevo milenio se ponen en vigencia mecanismos legales y programáticos que buscan responder al fenómeno multidimensional de la violencia donde, como se ha expuesto anteriormente, los procesos de debate a nivel internacional tuvieron relevante influencia tanto a nivel institucional como de reivindicación social y de denuncia.

Tabla N¹: Legislaciones vigentes Estados Parte MERCOSUR

\begin{tabular}{|c|c|c|c|}
\hline Argentina & Brasil & Paraguay & Uruguay \\
\hline \multicolumn{4}{|c|}{ Violencia contra las mujeres } \\
\hline 2010 & 2006 & 2016 & $2016 / 2018$ \\
\hline $\begin{array}{l}\text { Ley de protección } \\
\text { integral } \\
\text { prevenir, } \\
\text { sancionar } \\
\text { erradicar } \\
\text { violencia contra } \\
\text { las mujeres }\end{array}$ & $\begin{array}{l}\text { Ley María da } \\
\text { Penha. Contra la } \\
\text { violencia hacia } \\
\text { mujeres }\end{array}$ & $\begin{array}{l}\text { Ley de protección } \\
\text { integral a las } \\
\text { mujeres contra } \\
\text { toda forma de } \\
\text { violencia }\end{array}$ & $\begin{array}{l}\text { Plan de acción } \\
\text { contra la violencia } \\
\text { hacia las mujeres / } \\
\text { Ley que dicta } \\
\text { normas contra la } \\
\text { violencia hacia las } \\
\text { mujeres basada en } \\
\text { género }\end{array}$ \\
\hline \multicolumn{4}{|c|}{ Femicidio/feminicidio } \\
\hline 2012 & 2015 & 2016 & 2017 \\
\hline $\begin{array}{l}\text { Ley que tipifica } \\
\text { el homicidio } \\
\text { agravado de } \\
\text { mujeres }\end{array}$ & $\begin{array}{l}\text { Ley que tipifica el } \\
\text { feminicidio }\end{array}$ & 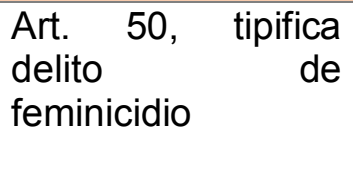 & $\begin{array}{ll}\text { Modificación } & \text { Art.312 } \\
\text { Código } & \text { Penal, } \\
\text { feminicidio } & \end{array}$ \\
\hline
\end{tabular}

Fuente: Elaboración propia a partir de información recabada de Observatorio de Igualdad de Género de América Latina y el Caribe (2019).

Tabla N²: Legislaciones vigentes Estados Parte SICA

\begin{tabular}{c|c|c} 
Belice & Costa Rica & El Salvador \\
& Violencia contra las mujeres &
\end{tabular}




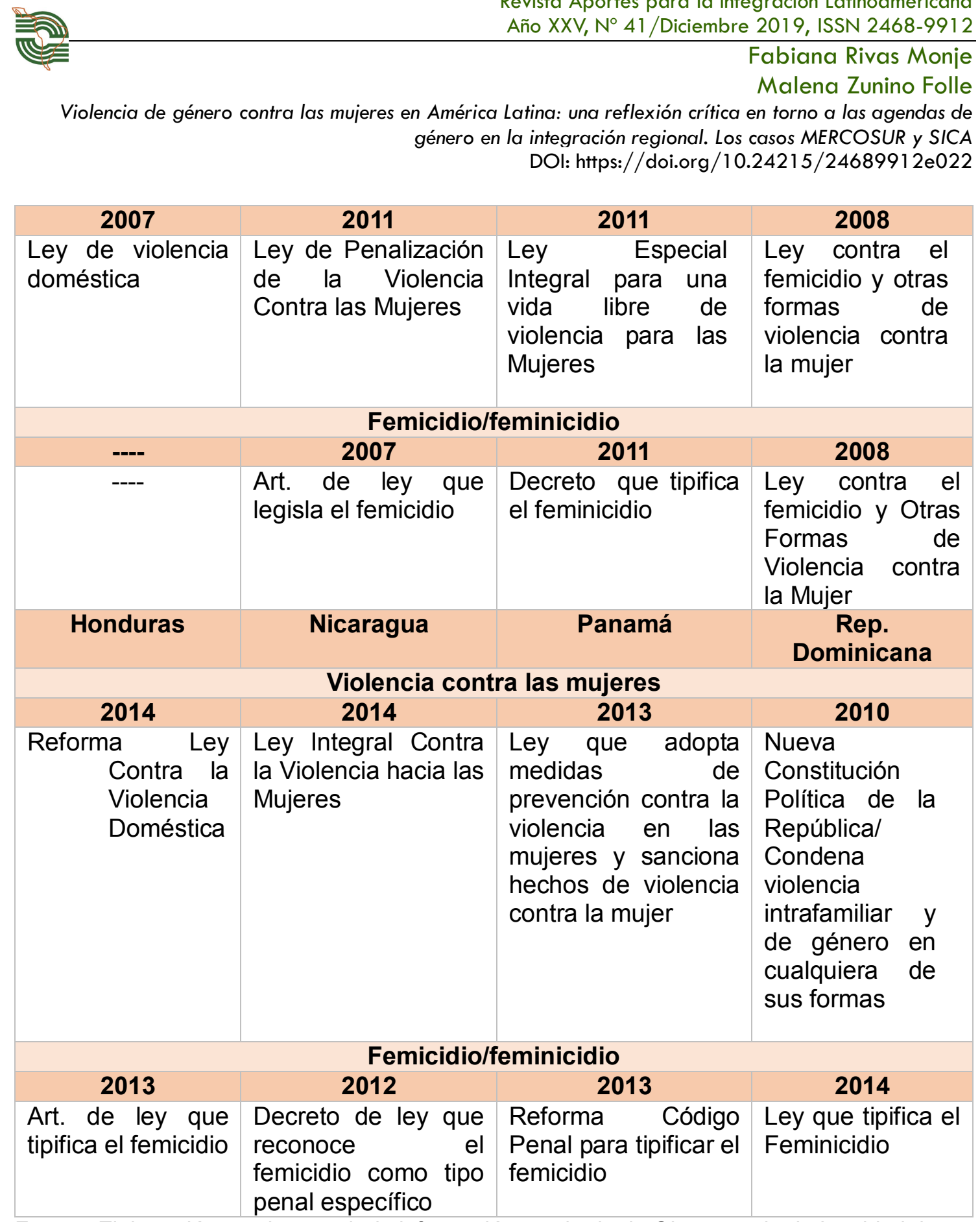

Fuente: Elaboración propia a partir de información recabada de Observatorio de Igualdad de Género de América Latina y el Caribe (2019).

En lo que respecta a estadísticas y, según el último informe de la $\mathrm{CEPAL}^{22}$, en 2017 al menos 2.795 mujeres fueron víctimas de feminicidio en 23 países de América Latina. En América del Sur la mayor prevalencia se observa en Bolivia y Paraguay, con tasas de 2.0 y 1.6 por cada 100.000 mujeres. En cuanto a los países del MERCOSUR y en números absolutos según las estadísticas oficiales, Argentina registró 251 feminicidios,

${ }^{22}$ Recuperado de: https://oig.cepal.org/es/indicadores/feminicidio 
Brasil 1133, Paraguay 53 y Uruguay 23. En términos generales, las situaciones menos alentadoras corresponden a los países de la región centroamericana. En este sentido, las tasas del 2016 - 2017 revelan que El Salvador $(10,2)$, Honduras $(5,8)$, Bélice $(4,8)$, Guatemala $(2,6)$ y República Dominicana $(2,2)$ son los países con mayor prevalencia de feminicidios (entre ellos se encuentra Trinidad y Tobago con una tasa de 3,0). Por otra parte, los números absolutos indican que durante 2017 se registraron 9 en Bélice, 26 en Costa Rica, 345 en El Salvador, 221 en Guatemala, 235 en Honduras, 67 en Nicaragua, 18 en Panamá y 119 en República Dominicana.

Como se puede observar, se han concretado avances en tipificación e identificación de la diversidad de tipos de violencia que se ejercen sobre las mujeres así como su carácter integral, complejo y multidimensional; se ha avanzado en la sistematización de datos y estadísticas regionales y nacionales al respecto; se conocen los problemas para denunciar, procesar y punir en los casos. Lo complejo es nominar la violencia (Rivas, 2017) y, también, lo casi imposible es desentrañarla de los hábitos arraigados de la vida comunitaria y familiar de todos los pueblos de mundo (Segato, 2002). En este sentido, si bien cada Estado Parte de ambos bloques ha integrado avances legislativos y estructurales en torno a la problemática, América Latina sigue siendo, según palabras de ONU, la "región más insegura y letal del mundo" para las mujeres, y estas acciones finalmente no se han traducido en una disminución de actos de violencia de género y su manifestación más extrema y visible como es el feminicidio, sino que la tendencia general va al aumento año a año.

A este respecto y, con la mira enfocada en la potencialidad de los procesos de integración regional, es evidente que en materia de género se han presentado avances importantes en términos de agenda y que erradicar las desigualdades fortalece la democracia y la ciudadanía contribuyendo al proceso mismo de cooperación e integración regional (Azar, Celiberti \& Espino, 2005). Sin embargo, cabe cuestionarse si es posible concretar de manera efectiva mecanismos que logren articular a los diversos niveles, así como actores sociales en pos de proceder a una erradicación progresiva de las violencias, considerando no sólo lo legislativo/punitivo, sino también los ámbitos de lo cultural, social, político, laboral, educativo, salud y vivienda, entre otros. Para ello, a nivel institucional debe asumirse una prioridad en las agendas y realizarse una transversalización eficiente de una perspectiva de género 
interseccional, es decir, que considere los cruces inseparables de la opresión de género con la clase, raza/etnia, sexualidad y otras dimensiones de dominación que determinan la experiencia social de las mujeres y que profundizan la desigualdad, la inequidad, la injusticia y la violencia, y así no recaer en la determinación de las políticas de género como de segunda categoría o focalizadas exclusivamente en mujeres.

Por otro lado, existe una evidente disparidad entre la responsabilidad encomendada a estas instancias -COMMCA y RMAMM, encargadas de la transversalización de la equidad de género en sus sistemas de integración regional-, y la capacidad real para llevar a cabo sus mandatos y funciones (ONU Mujeres, 2016). Aquí inciden diversos factores entre los que destacan el rango que ostentan dentro de sus bloques, así como la falta de consolidación, las reestructuraciones y reconfiguraciones que experimentan los organismos de integración, los cambios políticos internos a nivel de cada país y los cambios en la disponibilidad de recursos humanos, técnicos y financieros (ONU Mujeres, 2016). Las dificultades son diversas, pero el contexto social apremia y los desafíos están por delante, presentándose en distintos niveles donde se hace urgente actuar a través de las rutas más efectivas.

\section{Conclusiones}

A través de lo expuesto en torno a los procesos de integración regional observados con foco en las agendas de género de MERCOSUR y SICA, buscamos intencionar una lectura crítica respecto al lugar que ocupa y cómo es abordada la violencia de género contra las mujeres en América Latina. Consideramos que analizar dicho fenómeno a través de los lentes de la integración regional entrega elementos para problematizar, toda vez que vincular ambas dimensiones en pos de una superación de la preponderancia de lo económico y comercial. Al mismo tiempo nos permite poner de relieve la potencialidad y efectos que los procesos de integración regional pueden tener sobre el desarrollo social y humano a través de la promoción de la justicia y la equidad de género.

Si bien las desigualdades sociales por raza, etnia, clase y género que aquejan a las sociedades latinoamericanas no pueden explicarse sólo por las estructuras económicas, el funcionamiento de los mercados y economías como estructuras y 
procesos complejos, están íntimamente ligados a la perpetuación de estas desigualdades (Espino \& Underhill-Sem, 2012). En este sentido, es menester reconocer que han habido importantes avances progresivos en las agendas de los bloques respecto a la materia, como hemos venido desplegando, sin embargo, parecieran presentarse dificultades diversas y complejas que impiden que dichas instancias sean efectivas, a la vez que predomina un desconocimiento por parte de la sociedad civil del accionar concreto e incluso existencia de estas.

Parte de las posibles rutas de acción -respecto a la lucha por la equidad de género y contra las violencias- para los mecanismos de mujeres dentro de los bloques regionales abordados y, según recomendaciones de ONU Mujeres (2016), es la realización de procesos de reflexión interna respecto a estrategias de corto, mediano y largo plazo. También el análisis de posibles esfuerzos para un intercambio, complementariedad y articulación mutua con otros organismos e instancias de ámbito regional. Además, la identificación de estrategias de incidencia política en los sistemas de integración nacionales y la maximización del aprovechamiento de espacios de interlocución entre gobiernos ademas de la visibilización y fortalecimiento de mecanismos para facilitar la participación integral y sostenida de la sociedad civil a escala regional, logrando definir consultiva y conjuntamente prioridades donde las redes y organizaciones de mujeres y feministas cobran fundamental importancia.

Aquí creemos pertinente retomar las interrogantes planteadas por Ansaldi (2013), respecto a ¿desde dónde construir la integración latinoamericana?, ¿desde los niveles estado-céntricos, los gobiernos y/o Estados, o desde los pueblos?, ¿o desde unos y otros? Los resultados serán distintos si el rumbo es uno u otro, pues toda construcción desde arriba puede que sea más rápida pero sus bases serán débiles.

Construirla desde abajo es más lento, complejo y, por ende, más largo. Pero es la única forma de dotarla de basamento sólido. Cuanto más tiempo se tarde en seguir este camino, tanto más moroso será, ya no coronar el proceso, sino poner los cimientos. Por cierto, no es fácil decidir qué tipo de pozo hay que hacer para la construcción. Como en la de edificios, todo depende del suelo. En la metáfora, los suelos son las culturas, las memorias colectivas, los imaginarios sociales, la autoconciencia que los hombres y las mujeres de cada país latinoamericano han ido forjando a lo largo de su vida independiente. 
(Ansaldi, 2013, 26)

Los esfuerzos para transitar las rutas hacia sociedades justas, equitativas y libres de violencia deben dirigirse por estos rumbos, en la búsqueda constante de la superación de las asimetrías de poder y de primacía absoluta de los niveles estado-céntricos por sobre los pueblos. En los tiempos que hoy corren en América Latina, donde las cifras de violencia de género van en aumento y donde los colores políticos de los ejecutivos nacionales se tornan reaccionarios y conservadores, cabe interrogarse ¿qué efectos tendrá esto en los procesos de integración regional? Y, más concretamente, ¿qué consecuencias tendrá en las agendas de género de los bloques?; conjeturas no muy alentadoras pueden comenzar a urdirse desde ya. Hoy, parece hacerse más urgente que nunca tomar el desafío de una integración latinoamericana desde todas las amplias veredas de lo social.

\section{BiBLIOGRAFÍA}

Ansaldi, W. (2003). Por Patria entendemos la vasta extensión de ambas Américas. El proyecto de unidad latinoamericana en perspectiva histórica. Boletín de la Biblioteca del Congreso de la Nación, (127), (19-58). Buenos Aires, Argentina: Biblioteca del Congreso de la Nación. Recuperado de: https://bcn.gob.ar/labiblioteca/publicaciones/boletin-de-la-bcn

Azar, P.; Celiberti, L. \& Espino, A. (2005). La perspectiva de género en el marco de la integración regional en el MERCOSUR: hacia la construcción de una institucionalidad equitativa. Documento preparado para la Comisión Sectorial para el MercosurCOMISEC-Oficina de planeamiento y presupuesto, Foro Nacional de Organizaciones de Mujeres para la Integración y Mercosur. Recuperado de: https://www.ciedur.org.uy/adm/archivos/publicacion_83.pdf

Blanco Miranda, V. (2012). Apuntes críticos al Tratado Marco de Seguridad Democrática de Centro América. Económicas CUC, 33(1), 297-306. Recuperado de https://revistascientificas.cuc.edu.co/economicascuc/issue/view/31

Caballero, S. (2016). El desafío del mega-regionalismo: el multilateralismo en cuestión. Pensamiento Propio, (44), 77-106. Recuperado de: http://www.cries.org/?page id=33 
Consejo de Ministras de Centroamérica -Sistema de la Integración Centroamericana. (2013). Política Regional de Igualdad y Equidad de Género. Recuperado de https://www.sica.int/politicas/prieg

Espino, A. \& Underhill-Sem, Y. (2012). Género, equidad social y procesos económicos regionales: perspectivas de América Latina y el Pacífico. Development, 55(3), 358-368. Recuperado de: https://link.springer.com/journal/41301/55/3/page/1

Ferretto, M. \& Picasso, Y. (2018). La agenda de género en el MERCOSUR: logros y desafíos, ¿qué ocurre con los derechos sexuales y reproductivos?. Revista Electrónica Instituto de Investigaciones Jurídicas y Sociales Ambrosio L. Gioja, (20), 35-64. Recuperado de: http://www.derecho.uba.ar/revistas-digitales/index.php/revistaelectronica-gioja/issue/view/40

Gómez Castañeda, E. \& Naclerio Torres, L. (2011). "Mujer y Mercosur”: Una mirada retrospectiva y analítica sobre la incorporación de la agenda de género al proceso de integración regional". Revista Temas de Mujeres, (7), 163-189. Recuperado de: http://filo.unt.edu.ar/revista-temas-de-mujeres/

Jelin, E. (2002). Ciudadanía, movimientos sociales y Mercosur. Buenos Aires, Argentina: CONICET/UBA/IDES. Recuperado de: http://www.cid.unal.edu.co/cidnews/archivos/Linkstodocs/Ciudadania\%20Movimientos \%20Sociales $\% 20$ y 20 Mercosur.pdf

Letona, G. M. (2017). Participación política de las mujeres en Centroamérica: una mirada desde la integración regional. En J. P. Vargas Cespedes, E. T. Orantes Rivera \& P. Fernández Alarcon. (Comps.). Destino Centroamérica: Reflexiones para integrar la integración (pp. 384-421). La libertad, El Salvador: Ministerio de Asuntos exteriores y de Cooperación-Universidad Complutense Madrid-SICA. Recuperado de: https://www.academia.edu/37596545/PARTICIPACI\%C3\%93N POL\%C3\%8DTICA D E LAS MUJERES EN CENTROAM\%C3\%89RICA Una mirada desde la Integraci $\%$ C3\%B3n Regional

Malamud, A. (2011). Conceptos, teorías y debates sobre la integración regional. 
Norteamérica. Revista académica del CISAN-UNAM, 6(2), 219-249. Recuperado de: https://www.redalyc.org/articulo.oa?id=193722773008

Oficina Regional de Organización de las Naciones Unidas para Mujeres para las Américas y el Caribe Panamá. (2016). Documento técnico: Los mecanismos nacionales y regionales de las mujeres en América Latina y el Caribe Hispano. Recuperado de: https://lac.unwomen.org/es/digiteca/publicaciones/2016/mecanismosnacionales-y-regionales-de-la-mujer

Reunión de Ministras y Altas Autoridades de la Mujer del Mercosur. (2014). Política de Igualdad de Género del Mercosur. Directrices. Uruguay: INMUJERES. http://guiaderecursos.mides.gub.uy/innovaportal/file/34842/1/directrices_12nov2014.pd $f$

Rivas Monje, F. (2017). Las limitaciones teóricas respecto a la violencia de género contra las mujeres: aportes desde el feminismo descolonial para el análisis en mujeres de América Latina. Iberoamérica Social: revista red de estudios sociales, (7), 129-153. Recuperado de: https://iberoamericasocial.com/las-limitaciones-teoricas-respecto-laviolencia-genero-las-mujeres-aportes-desde-feminismo-descolonial-analisis-mujeresamerica-latina/

Sánchez Pérez, R. (2017). El análisis de las dinámicas de interlocución de la sociedad civil en el marco del Acuerdo de SICA-UE. En J. P. Vargas Céspedes, E. T. Orantes Rivera \& P. Fernández Alarcón. (Comps.). Destino Centroamérica: Reflexiones para integrar la integración (pp. 355-383). La libertad, El Salvador: Ministerio de Asuntos exteriores y de Cooperación-Universidad Complutense Madrid-SICA. Recuperado de: https://www.academia.edu/35404563/Destino Centroam\%C3\%A9rica reflexiones par a integrar la integraci\%C3\%B3n

Santos Carrillo, F. (2016). Los modos de las políticas de la Integración Centroamericana. Revista de Derecho, (20), 31-46. https://doi.org/10.5377/derecho.v0i20.2787

Segato, R. (2003). Las estructuras de la violencia: contrato y status en la etiología de 

género en la integración regional. Los casos MERCOSUR y SICA DOI: https://doi.org/10.24215/2468991 2e022

la violencia. Serie Antropología, (334), 1-19. Recuperado de: http://dan.unb.br/images/doc/Serie334empdf.pdf

Van Klaveren, A. (1990). Las relaciones internacionales de América Latina en la década de 1980: cambio y continuidad. Estudios Internacionales, 23(89) 82-118. https://revistaei.uchile.cl/index.php/REl/article/view/15552

Van Klaveren, A. (1997). Regionalismo y multilateralismo: una convergencia necesaria. En S. López Ayllon (Coord.) El Futuro del libre comercio en el continente americano análisis y perspectivas (pp. 51-69). México: Instituto de Investigaciones Jurídicas UNAM. Recuperado de: https://archivos.juridicas.unam.mx/www/bjv/libros/1/143/6.pdf

Wionczek, M. (1970). Surgimiento y decadencia de la integración económica latinoamericana". Foro internacional, 11(41), 1-18. Recuperado de: https://forointernacional.colmex.mx/index.php/fi/issue/view/40/showToc

RIvAS MONJE, FabiANA: Socióloga, Universidad de La Frontera, Temuco, Chile. Maestranda en
Estudios Sociales Latinoamericanos por la Facultad de Ciencias Sociales de la Universidad de
Buenos Aires. Correo electrónico: fabiana.rivas.monje@gmail.com.
Zunino FolLE, MalenA:. Socióloga, Facultad de Ciencias Sociales-Universidad de la
República, Uruguay. Maestranda en Estudios Sociales Latinoamericanos por la Facultad de
Ciencias Sociales de la Universidad de Buenos Aires. Correo electónico:
malenazunino@gmail.com.

